\title{
Some Results of Ruin Probability for the Classical Risk Process ${ }^{\dagger}$
}

\author{
HE YUANJIANG \\ Department of Statistics Science, Zhongshan University, Ganglion, 510275 PRChina \\ LI XUCHENG \\ Department of Statistics Science, Zhongshan University, Ganglion, 510275 PRChina \\ JOHN ZHANG* \\ Department of Mathematics, Indiana University of Pennsylvania, Indiana PA, 15705, \\ USA
}

\begin{abstract}
The computation of ruin probability is an important problem in the collective risk theory. It has applications in the fields of insurance, actuarial science, and economics. Many mathematical models have been introduced to simulate business activities and ruin probability is studied based on these models. Two of these models are the classical risk model and the Cox model. In the classical model, the counting process is a Poisson process and in the Cox model, the counting process is a Cox process. Thorin (1973) studied the ruin probability based on the classical model with the assumption that random sequence followed the $\Gamma$ distribution with density function $f(x)=\frac{x^{\frac{1}{\beta}-1}}{\beta^{\frac{1}{\beta}} \Gamma(1 / \beta)} e^{-\frac{x}{\beta}}, x>0$, where $\beta>1$. This paper studies the ruin probability of the classical model where the random sequence follows the $\Gamma$ distribution with density function $f(x)=\frac{\alpha^{n}}{\Gamma(n)} x^{n-1} e^{-\alpha x}, \quad x>0$, where $\alpha>0$ and $n \geq 2$ is a positive integer. An intermediate general result is given and a complete solution is provided for $n=2$. Simulation studies for the case of $n=2$ is also provided.
\end{abstract}

Keywords: Collective risk theory; Gamma distribution; Ruin probability; Simulation

\section{Introduction}

To study the probability of ruin, many models have been proposed and investigated. These models include the classical and the Cox model. Although the applications of ruin probability are not restricted to the insurance type problem, the idea can be explained easily by an insurance business model. An insurance company's capital at time $t$ can be expressed as

$\dagger$ Supported in part by National Natural Science Foundation of China and China Bridges International

* Requests for reprints should be sent to John Zhang, Department of Mathematics, Indiana University of Pennsylvania 233 Stright Hall, Indiana PA, 15705, USA. 
$R(t)=u+C(t)-O(t)$, where $R(t)$ is the company's capital at time $t, u$ is the initial capital, $C(t)$ is the income function, and $O(t)$ is the expense function. The income function $C(t)$ can be expressed as a relatively simple function and often taken to be $C(t)=c t$, where $c>0$ is the income coefficient. The expense function $O(t)$, however, is a random process because of the nature of the insurance claims. One way to describe $O(t)$ is to define:

$$
O(t)=\sum_{i=1}^{N(t)} Z_{i},
$$

where $Z_{i}$ is the size of the payment and $N(t)$ is a counting process. The classical model assumes that $N(t)$ is a Poisson process and the Cox model assumes that $N(t)$ is a Cox process. Thorin (1973) studied the classical model where $Z_{i}$ has $\Gamma$ distribution with density function

$$
f(x)=\frac{x^{\frac{1}{\beta}-1}}{\beta^{\frac{1}{\beta}} \Gamma(1 / \beta)} e^{-\frac{x}{\beta}}, \quad x>0,
$$

where $\beta>1$. In this paper, we study the classical model when $Z_{i}$ follows the $\Gamma$ distribution with density function

$$
f(x)=\frac{\alpha^{n}}{\Gamma(n)} x^{n-1} e^{-\alpha x}, \quad x>0,
$$

where $\alpha>0$ and $n \geq 2$ is a positive integer.

Thorin's case models situations where the probability distribution of ten random payments $Z_{i}$ is monotonically strictly decrease. The case we study here can be applied to situations when the probability distribution of the random payments $Z_{i}$ places higher mass to values near the center. It is meaningful to study this case because many data sets in practice have mount shape distributions.

The organization of the paper is as follows: In section 2, we give the main theorems and section 3 presents the simulation study. Section 4 contains the concluding remarks.

\section{Main Results}

We define a risk process as

$$
X(t)=c t-\sum_{k=1}^{N(t)} Z_{k},
$$


where $N(t)$ is a counting process, $\left\{Z_{k}\right\}_{1}^{\infty}$ is a sequence of independent random variables with identical distributions. We further assume that the common distribution function is $F$ with mean $\mu$ and variance $\sigma^{2}$, and $F(0)=0$. The counting process $N(t)$ and $\left\{Z_{k}\right\}_{1}^{\infty}$ are independent, and $c$ is a positive constant.

$N(t)$ can be interpreted as the number of claims of an insurance company in the time interval $(0, t]$. At each jump point of $N(t)$, the insurance company has to pay out a stochastic amount of money. On the other hand, the company receives $c$ units of revenue per unit time.

Let $\Psi(u)=P\{u+X(t)<0$ for some $t>0\}$. Thenfor $u \geq 0, \Psi(u)$ can be interpreted as the ruin probability of an insurance company which has initial capital $u$ when facing the risk process $X(t) . \Phi(u)=1-\Psi(u)$ is the non-ruin probability. Notice that $\Psi(u)=1$ for $u<0$.

From now on, we assume that $N(t)$ is a Poisson process with standing $\lambda>0$, and therefore, $E(N(t))=\lambda t$. Let

$$
\rho=\frac{c-\lambda \mu}{\lambda \mu} .
$$

Then

$$
E X(t)=3 D c t-E \sum_{i=3 D 1}^{N(t)} Z_{i}=3 D c t-E N(t) E Z_{1}=3 D c t-\lambda \mu t=\rho \lambda \mu t .
$$

We can see that

$$
\rho=\frac{E X(t)}{\lambda \mu t},
$$

and is called the safety loading. It is the ratio of the expected profit to the expected payments. $\rho=1$, for example, indicates that the company's expected profit is of the same size as its expected payments. Larger value of $\rho$ indicates a company is in a "save" state. Thus $\rho$ can be considered as an index to measure the safety (non-ruin) of an insurance company.

We also assume that

$$
\rho>0 .
$$

This condition can be interpreted as the premiums received per unit time exceed the expected claim payments per unit time.

If $Z_{i}$ has exponential distribution, then

$$
\Psi(u)=\frac{1}{1+\rho} e^{-\frac{\rho u}{\mu(1+\rho)}}
$$


(for example, see Grandell(1991)). If $Z_{i}$ has $\Gamma$ distribution with density function

$$
f(x)=\frac{x^{\frac{1}{\beta}-1}}{\beta^{\frac{1}{\beta}} \Gamma(1 / \beta)} e^{-\frac{x}{\beta}}, \quad x>0,
$$

where $\beta>1$, then the ruin probability is

$\Psi(u)=\frac{\rho(1-\beta R) e^{-R u}}{1+(1+\rho)(R+R \beta-1)}+\frac{\rho}{\pi \beta} \sin \frac{\pi}{\beta} \int_{0}^{\infty} \frac{x^{\frac{1}{\beta}} e^{-(x+1) u / \beta} d x}{\left\{x^{\frac{1}{\beta}}\left[1+(1+\rho) \frac{x+1}{\beta}\right]-\cos \frac{\pi}{\beta}\right\}^{2}+\sin ^{2} \frac{\pi}{\beta}}$,

where $R$ is the positive solution of the equation

$$
\int_{0}^{+\infty} e^{r z} d F(z)-1=\frac{c r}{\lambda}
$$

for $r<1 / \beta$, that is, the positive solution of the equation

$$
(1-\beta r)^{-\frac{1}{\beta}}-1=(1+\rho) r
$$

for $r<1 / \beta$ (for example, see Thorin (1973),or Grandell (1991), p.14).

Now we consider the case that $Z_{i}$ has $\Gamma$ distribution with density function

$$
f(x)=\frac{\alpha^{n}}{\Gamma(n)} x^{n-1} e^{-\alpha x}, \quad x>0,
$$

where $\alpha>0$ and $n$ is a positive integer.

We state a well-known result as a lemma:

Lemma 1 For a classical risk model, if $N(t)$ has intensity $\lambda$ and $Z_{i}$ has distribution function $F(z)$, then

$$
D \Phi(u)=\frac{\lambda}{c} \Phi(u)-\frac{\lambda}{c} \int_{0}^{u} \Phi(u-z) d F(z) .
$$

Here, $D=\frac{d}{d u}$ is the differential operator.

(Interested readers please see equation (3) in Grandell (1991), p.4.)

Theorem 1 For a classical risk model, if $N(t)$ has intensity $\lambda$ and $Z_{i}$ has $\Gamma$ distribution with density function

$$
f(x)=\frac{\alpha^{n}}{\Gamma(n)} x^{n-1} e^{-\alpha x}, \quad x>0,
$$

where $\alpha>0$ and $n \geq 1$ is an integer, then the non-ruin probability $\Phi(u)$ is a solution of the ordinary differential equation:

$$
D(D+\alpha)^{n} \Phi(u)-\frac{\lambda}{c}(D+\alpha)^{n} \Phi(u)+\frac{\lambda}{c} \alpha^{n} \Phi(u)=0 .
$$


Proof. From Lemma 1, we have

$$
D \Phi(u)=\frac{\lambda}{c} \Phi(u)-\frac{\lambda}{c} \int_{0}^{u} \Phi(u-z) d F(z) .
$$

Substituting $d F(z)=f(z) d z$, we have

$$
D \Phi(u)=\frac{\lambda}{c} \Phi(u)-\frac{\lambda}{c} \int_{0}^{u} \Phi(u-z) \frac{\alpha^{n}}{\Gamma(n)} z^{n-1} e^{-\alpha z} d z .
$$

The change of variables $z=u-w$ leads to

$$
D \Phi(u)=\frac{\lambda}{c} \Phi(u)-\frac{\lambda}{c} \int_{0}^{u} \Phi(w) \frac{\alpha^{n}}{\Gamma(n)}(u-w)^{n-1} e^{-\alpha(u-w)} d w .
$$

Let $A(u ; 0)=\Phi(u)$ and let

$$
A(u ; k)=\int_{0}^{u} \Phi(w) \frac{\alpha^{k}}{\Gamma(k)}(u-w)^{k-1} e^{-\alpha(u-w)} d w
$$

for $1 \leq k \leq n$.

By $(2), \Phi(w)$ is differentiable. Let $h(w, \eta)=\Phi(w) \alpha e^{-\alpha(\eta-w)}$. Then $h(w, \eta)$ and $\frac{\partial h(w, \eta)}{\partial \eta}$ are continuous. Let

$$
g(\xi(u), \eta(u))=\int_{0}^{\xi(u)} \Phi(w) \alpha e^{-\alpha(\eta(u)-w)} d w, \quad \xi(u)=u, \quad \eta(u)=u .
$$

Then by Lang (1979), p.119, Theorem 5 of Chapter V, we can exchange the order of differentiation and integration, so we have

$$
\begin{aligned}
D A(u ; 1)= & D g(\xi, \eta) \\
= & \frac{\partial g}{\partial \xi} \frac{d \xi}{d u}+\frac{\partial g}{\partial \eta} \frac{d \eta}{d u} \\
= & \alpha \Phi(\xi) e^{-\alpha(\eta-\xi)} \\
& \quad-\alpha \int_{0}^{\xi} \Phi(w) \alpha e^{-\alpha(\eta-w)} d w \\
= & \alpha \Phi(u)-\alpha \int_{0}^{u} \Phi(w) \alpha e^{-\alpha(u-w)} d w \\
= & \alpha A(u ; 0)-\alpha A(u ; 1) .
\end{aligned}
$$


And by using similar methods, for $2 \leq k \leq n$, we have

$$
\begin{aligned}
& D A(u ; k) \\
= & (k-1) \int_{0}^{u} \Phi(w) \frac{\alpha^{k}}{\Gamma(k)}(u-w)^{k-2} e^{-\alpha(u-w)} d w \\
& \quad-\alpha \int_{0}^{u} \Phi(w) \frac{\alpha^{k}}{\Gamma(k)}(u-w)^{k-1} e^{-\alpha(u-w)} d w \\
= & \alpha A(u ; k-1)-\alpha A(u ; k) .
\end{aligned}
$$

So for $1 \leq k \leq n$, we have

$$
\frac{D+\alpha}{\alpha} A(u ; k)=A(u ; k-1) .
$$

Thus

$$
\left(\frac{D+\alpha}{\alpha}\right)^{n} A(u ; n)=A(u ; 0)=\Phi(u) .
$$

From (3) and (4), we have

$$
D \Phi(u)=\frac{\lambda}{c} \Phi(u)-\frac{\lambda}{c} A(u ; n) .
$$

From (5) and (6), we have

$$
\begin{aligned}
D\left(\frac{D+\alpha}{\alpha}\right)^{n} \Phi(u) & =\left(\frac{D+\alpha}{\alpha}\right)^{n} D \Phi(u) \\
& =\frac{\lambda}{c}\left(\frac{D+\alpha}{\alpha}\right)^{n} \Phi(u)-\frac{\lambda}{c}\left(\frac{D+\alpha}{\alpha}\right)^{n} A(u ; n) \\
& =\frac{\lambda}{c}\left(\frac{D+\alpha}{\alpha}\right)^{n} \Phi(u)-\frac{\lambda}{c} \Phi(u)
\end{aligned}
$$

So

$$
D(D+\alpha)^{n} \Phi(u)-\frac{\lambda}{c}(D+\alpha)^{n} \Phi(u)+\frac{\lambda}{c} \alpha^{n} \Phi(u)=0 .
$$

Remark. If $n=1$, equation (1) becomes

$$
D(D+\alpha) \Phi(u)-\frac{\lambda}{c}(D+\alpha) \Phi(u)+\frac{\lambda \alpha}{c} \Phi(u)=0 .
$$

After simplification, we have

$$
D^{2} \Phi(u)+\left(\alpha-\frac{\lambda}{c}\right) D \Phi(u)=0,
$$


or

$$
D^{2} \Phi(u)=-\frac{\alpha \rho}{1+\rho} D \Phi(u) .
$$

The above equation is solved in Grandell (1991), page 6.

The following theorem gives a complete solution of differential equation (1) for $n=2$.

Theorem 2 For a classical risk model, if $N(t)$ has intensity $\lambda$ and $Z_{i}$ has $\Gamma$ distribution with density function

$$
f(x)=\frac{\alpha^{2}}{\Gamma(2)} x e^{-\alpha x}, x>0,
$$

then the non-ruin probability $\Phi(u)$ is :

$$
\Phi(u)=1+\frac{\nu_{2}\left(\nu_{1}+\alpha\right)^{2}}{\left(\nu_{1}-\nu_{2}\right) \alpha^{2}} e^{\nu_{1} u}+\frac{\nu_{1}\left(\nu_{2}+\alpha\right)^{2}}{\left(\nu_{2}-\nu_{1}\right) \alpha^{2}} e^{\nu_{2} u},
$$

where

$$
\begin{aligned}
& \nu_{1}=\frac{\lambda-2 c \alpha+\sqrt{\lambda^{2}+4 c \alpha \lambda}}{2 c}, \\
& \nu_{2}=\frac{\lambda-2 c \alpha-\sqrt{\lambda^{2}+4 c \alpha \lambda}}{2 c} .
\end{aligned}
$$

Proof. By Theorem 1, $\Phi(u)$ is a solution of the ordinary differential equation:

$$
D(D+\alpha)^{2} \Phi(u)-\frac{\lambda}{c}(D+\alpha)^{2} \Phi(u)+\frac{\lambda}{c} \alpha^{2} \Phi(u)=0 .
$$

The characteristic equation

$$
x(x+\alpha)^{2}-\frac{\lambda}{c}(x+\alpha)^{2}+\frac{\lambda}{c} \alpha^{2}=0
$$

can be rewritten as

$$
x\left[x^{2}+\left(2 \alpha-\frac{\lambda}{c}\right) x+\left(\alpha^{2}-\frac{2 \alpha \lambda}{c}\right)\right]=0,
$$

and has solutions

$$
\begin{aligned}
& \nu_{0}=0, \\
& \nu_{1}=\frac{\lambda-2 c \alpha+\sqrt{\lambda^{2}+4 c \alpha \lambda}}{2 c}, \\
& \nu_{2}=\frac{\lambda-2 c \alpha-\sqrt{\lambda^{2}+4 c \alpha \lambda}}{2 c} .
\end{aligned}
$$


And (7) has general solution

$$
\Phi(u)=c_{0}+c_{1} e^{\nu_{1} u}+c_{2} e^{\nu_{2} u} .
$$

Since

$$
\rho=\frac{c-\lambda \mu}{\lambda \mu}>0, \quad \mu=\frac{2}{\alpha}
$$

we have

$$
\frac{c \alpha-2 \lambda}{2 \lambda}>0
$$

So

$$
\alpha>\frac{2 \lambda}{c}
$$

and hence

$$
2 \alpha>\alpha>\frac{2 \lambda}{c}>\frac{\lambda}{c}
$$

Thus

$$
\alpha^{2}-\frac{2 \alpha \lambda}{c}>0 \text { and } 2 \alpha-\frac{\lambda}{c}>0 .
$$

Since $\nu_{1}$ and $\nu_{2}$ are the solutions of equation (9), we have

$$
\nu_{1}<0 \text { and } \nu_{2}<0 \text {. }
$$

Thus, let $u \rightarrow \infty$, we have:

$$
c_{0}=\Phi(\infty)=1
$$

From (3), (10) and (11), we have

$$
\begin{aligned}
& c_{1} \nu_{1} e^{\nu_{1} u}+c_{2} \nu_{2} e^{\nu_{2} u} \\
= & \frac{\lambda}{c}\left(1+c_{1} e^{\nu_{1} u}+c_{2} e^{\nu_{2} u}\right)-\frac{\lambda}{c} \int_{0}^{u}\left(1+c_{1} e^{\nu_{1} w}+c_{2} e^{\nu_{2} w}\right) \frac{\alpha^{2}}{\Gamma(2)}(u-w) e^{-\alpha(u-w)} d w .
\end{aligned}
$$

After simplification, we have

$$
\begin{aligned}
& {\left[\nu_{1}-\frac{\lambda}{c}+\frac{\lambda \alpha^{2}}{c\left(\nu_{1}+\alpha\right)^{2}}\right] c_{1} e^{\nu_{1} u}+\left[\nu_{2}-\frac{\lambda}{c}+\frac{\lambda \alpha^{2}}{c\left(\nu_{2}+\alpha\right)^{2}}\right] c_{2} e^{\nu_{2} u} } \\
= & \frac{\lambda}{c} e^{-\alpha u}\left\{(\alpha u+1)+\alpha^{2}\left\{\frac{c_{1}}{\left(\nu_{1}+\alpha\right)^{2}}\left[\left(\nu_{1}+\alpha\right) u+1\right]+\right.\right. \\
& \left.\left.\frac{c_{2}}{\left(\nu_{2}+\alpha\right)^{2}}\left[\left(\nu_{2}+\alpha\right) u+1\right]\right\}\right\} .
\end{aligned}
$$


From (8), we have

$$
\nu_{1}-\frac{\lambda}{c}+\frac{\lambda \alpha^{2}}{c\left(\nu_{1}+\alpha\right)^{2}}=0
$$

and

$$
\nu_{2}-\frac{\lambda}{c}+\frac{\lambda \alpha^{2}}{c\left(\nu_{2}+\alpha\right)^{2}}=0
$$

So

$$
\alpha u+1+\alpha^{2}\left\{\frac{c_{1}}{\left(\nu_{1}+\alpha\right)^{2}}\left[\left(\nu_{1}+\alpha\right) u+1\right]+\frac{c_{2}}{\left(\nu_{2}+\alpha\right)^{2}}\left[\left(\nu_{2}+\alpha\right) u+1\right]\right\}=0,
$$

that is,

$$
\left(\alpha+\frac{\alpha^{2} c_{1}}{\nu_{1}+\alpha}+\frac{\alpha^{2} c_{2}}{\nu_{2}+\alpha}\right) u+\frac{\alpha^{2} c_{1}}{\left(\nu_{1}+\alpha\right)^{2}}+\frac{\alpha^{2} c_{2}}{\left(\nu_{2}+\alpha\right)^{2}}+1=0 .
$$

Therefore we have

$$
\alpha+\frac{\alpha^{2} c_{1}}{\nu_{1}+\alpha}+\frac{\alpha^{2} c_{2}}{\nu_{2}+\alpha}=0
$$

and

$$
\frac{\alpha^{2} c_{1}}{\left(\nu_{1}+\alpha\right)^{2}}+\frac{\alpha^{2} c_{2}}{\left(\nu_{2}+\alpha\right)^{2}}+1=0
$$

The solution is

$$
c_{1}=\frac{\nu_{2}\left(\nu_{1}+\alpha\right)^{2}}{\left(\nu_{1}-\nu_{2}\right) \alpha^{2}}, \quad c_{2}=\frac{\nu_{1}\left(\nu_{2}+\alpha\right)^{2}}{\left(\nu_{2}-\nu_{1}\right) \alpha^{2}} .
$$

Thus we have

$$
\Phi(u)=1+\frac{\nu_{2}\left(\nu_{1}+\alpha\right)^{2}}{\left(\nu_{1}-\nu_{2}\right) \alpha^{2}} e^{\nu_{1} u}+\frac{\nu_{1}\left(\nu_{2}+\alpha\right)^{2}}{\left(\nu_{2}-\nu_{1}\right) \alpha^{2}} e^{\nu_{2} u} .
$$

Theorem 2 has an interesting equivalent version. It is stated as follows:

Theorem 3 For a classical risk model, if $N(t)$ has intensity $\lambda=1$ and $Z_{i}$ has $\Gamma$ distribution with density function

$$
f(x)=\frac{1}{\Gamma(2)} x e^{-x}, \quad x>0,
$$

then the non-ruin probability $\Phi(u)$ is: 


$$
\Phi(u)=1+\frac{\nu_{2}\left(\nu_{1}+1\right)^{2}}{\nu_{1}-\nu_{2}} e^{\nu_{1} u}+\frac{\nu_{1}\left(\nu_{2}+1\right)^{2}}{\nu_{2}-\nu_{1}} e^{\nu_{2} u},
$$

where

$$
\begin{aligned}
& \nu_{1}=\frac{1-2 c+\sqrt{1+4 c}}{2 c}, \\
& \nu_{2}=\frac{1-2 c-\sqrt{1+4 c}}{2 c} .
\end{aligned}
$$

It is obvious that Theorem 2 implies Theorem 3 (by letting $\lambda=1, \alpha=1$ ). Now we show that Theorem 3 implies Theorem 2 as follows:

Suppose that Theorem 3 holds. For a classical risk model, let $N(t)$ be a Poisson process with intensity $\lambda$, and assume all $Z_{i}$ 's have $\Gamma$ distribution with identical density function

$$
f(z)=\frac{\alpha^{2}}{\Gamma(2)} z e^{-\alpha z}, z>0 .
$$

Let

$$
\tilde{N}(t)=N(t / \lambda)
$$

and

$$
\tilde{Z}_{i}=\alpha Z_{i}
$$

for all $i$. Then $\tilde{N}(t)$ is a Poisson process with intensity 1 , all $\tilde{Z}_{i}$ s have identical density function

$$
\tilde{f}(\tilde{z})=\frac{1}{\Gamma(2)} \tilde{z} e^{-\tilde{z}}, \tilde{z}>0 .
$$

Let

$$
R(t)=u+c t-\sum_{i=1}^{N(t)} Z_{i}
$$

and

$$
\tilde{R}(t)=\tilde{u}+\tilde{c} t-\sum_{i=1}^{\tilde{N}(t)} \tilde{Z}_{i}
$$

where

$$
\tilde{u}=\alpha u, \quad \tilde{c}=\frac{\alpha c}{\lambda} .
$$


Then

$$
\tilde{R}(\lambda t)=\alpha u+\frac{c \alpha}{\lambda} \cdot \lambda t-\alpha \sum_{i=1}^{\tilde{N}(\lambda t)} Z_{i}=\alpha u+\alpha c t-\alpha \sum_{i=1}^{N(t)} Z_{i}=\alpha R(t) .
$$

Let

$$
\left.\begin{array}{ccc}
\Phi(u)=1-P\{R(t)<0 & \text { for some } & t \\
\tilde{\Phi}(\tilde{u})=1-P\{\tilde{R}(t)<0 & \text { for some } & t
\end{array}\right\} .
$$

Then by (13) and Theorem 3 we have

$$
\Phi(u)=\tilde{\Phi}(\tilde{u})=1+\frac{\nu_{2}\left(\nu_{1}+1\right)^{2}}{\nu_{1}-\nu_{2}} e^{\nu_{1} \tilde{u}}+\frac{\nu_{1}\left(\nu_{2}+1\right)^{2}}{\nu_{2}-\nu_{1}} e^{\nu_{2} \tilde{u}},
$$

where

$$
\begin{aligned}
\nu_{1} & =\frac{1-2 \tilde{c}+\sqrt{1+4 \tilde{c}}}{2 \tilde{c}}, \\
\nu_{2} & =\frac{1-2 \tilde{c}-\sqrt{1+4 \tilde{c}}}{2 \tilde{c}} .
\end{aligned}
$$

It is easy to see that Theorem 2 comes from (14) if we replace $\tilde{u}$ and $\tilde{c}$ by $\alpha u$ and $\alpha c / \lambda$, respectively.

\section{Simulation Study}

For the risk process in Theorem 3, we conducted a simulation study to demonstrate the relationships between the non-ruin probability, the initial capital and the revenue coefficient c. The simulation is carried out using SAS version 6.12 on a PC with CPU PII-300. In example 1, we simulate the risk process 1000000 times, while in example 2, for each case (observation 1-18) we simulate the risk process 10000 times. The following is part of the program and the results:

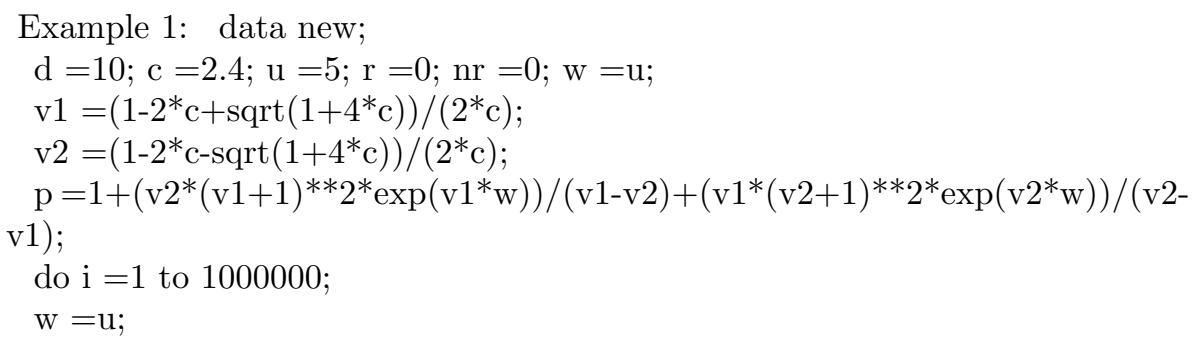


$\mathrm{k}: \mathrm{w}=\mathrm{w}+\mathrm{c}^{*}$ ranexp(1)-rangam $(\mathrm{d}, 2)$;

if $\mathrm{w}>=0$ and $\mathrm{w}<=500$ then goto $\mathrm{k}$;

if $\mathrm{w}>500$ then $\mathrm{nr}=\mathrm{nr}+1$;

if $\mathrm{w}<0$ then $\mathrm{r}=\mathrm{r}+1$;

end;

$\mathrm{f}=\mathrm{nr} /(\mathrm{r}+\mathrm{nr})$;

$\mathrm{e}=\mathrm{f}-\mathrm{p}$;

$\mathrm{s}=1000 * \mathrm{e} / \operatorname{sqrt}\left(\mathrm{p}^{*}(1-\mathrm{p})\right)$;

proc print;

var c u nr f p e s;

run;

$$
\begin{array}{cccccccc}
\text { OBS } & \mathrm{C} & \mathrm{U} & \mathrm{NR} & \mathrm{F} & \mathrm{P} & \mathrm{E} & \mathrm{S} \\
1 & 2.4 & 5 & 516550 & 0.51655 & 0.51681 & -.00026197 & -0.52424
\end{array}
$$

NOTE: The DATA statement used 4 hours 14 minutes 33.72seconds.

Example 2:

\begin{tabular}{rrrrrcrr} 
OBS & $\mathrm{C}$ & $\mathrm{U}$ & $\mathrm{NR}$ & $\mathrm{F}$ & $\mathrm{P}$ & $\mathrm{E}$ & \multicolumn{1}{c}{$\mathrm{S}$} \\
1 & 2.1 & 3 & 1350 & 0.1350 & 0.12984 & 0.0051617 & 1.53565 \\
2 & 2.1 & 5 & 1803 & 0.1803 & 0.18360 & -.0032976 & -0.85176 \\
3 & 2.1 & 10 & 3027 & 0.3027 & 0.30402 & -.0013219 & -0.28738 \\
4 & 2.1 & 50 & 8043 & 0.8043 & 0.80586 & -.0015609 & -0.39464 \\
5 & 2.1 & 100 & 9588 & 0.9588 & 0.96064 & -.0018441 & -0.94842 \\
6 & 2.1 & 200 & 9985 & 0.9985 & 0.99838 & 0.0001173 & 0.29203 \\
7 & 2.2 & 3 & 2302 & 0.2302 & 0.23523 & -.0050277 & -1.18538 \\
8 & 2.2 & 5 & 3197 & 0.3197 & 0.32330 & -.0036049 & -0.77071 \\
9 & 2.2 & 10 & 5021 & 0.5021 & 0.50181 & 0.0002863 & 0.05727 \\
10 & 2.2 & 50 & 9565 & 0.9565 & 0.95701 & -.0005116 & -0.25223 \\
11 & 2.2 & 100 & 9979 & 0.9979 & 0.99799 & -.0000895 & -0.19984 \\
12 & 2.2 & 200 & 10000 & 1.0000 & 1.00000 & 0.0000044 & 0.20970 \\
13 & 2.4 & 3 & 3911 & 0.3911 & 0.39403 & -.0029332 & -0.60029 \\
14 & 2.4 & 5 & 5223 & 0.5223 & 0.51681 & 0.0054880 & 1.09823 \\
15 & 2.4 & 10 & 7321 & 0.7321 & 0.72589 & 0.0062069 & 1.39148 \\
16 & 2.4 & 50 & 9969 & 0.9969 & 0.99706 & -.0001606 & -0.29658 \\
17 & 2.4 & 100 & 10000 & 1.0000 & 0.99999 & 0.0000101 & 0.31849 \\
18 & 2.4 & 200 & 10000 & 1.0000 & 1.00000 & 0.0000000 & 0.00110
\end{tabular}

Here, $\mathrm{C}$ and $\mathrm{U}$ are the $\mathrm{c}$ and $\mathrm{u}$ in Theorem 3, respectively. (Recall that $\mathrm{u}$ is the initial capital of an insurance company and the company receives c units of revenue per unit time.) $\mathrm{P}$ is the non-ruin probability $\Phi(u)$ in 
Theorem 3 calculated by using equation (12); NR is the number of the non-ruin risk processes; $\mathrm{F}$ is the relative frequency of non-ruin, and $\mathrm{E}$ is the difference of $\mathrm{F}$ and $\mathrm{P}$. E can be interpreted as the error of simulation results (assuming our theory is correct.) The small $\mathrm{E}$ values in our study confirm our theoretical results from a different perspective.

For the estimation of the ruin probability $\Psi(u)$, we can use the Lundberg inequality

$$
\Psi(u) \leq e^{-R u},
$$

where $R$ is the Lundberg exponent, that is, $R$ is the positive solution of the equation

$$
\int_{0}^{+\infty} e^{r z} d F(z)-1=\frac{c r}{\lambda}
$$

(For example, see Grandell, p.11.) For the risk process in Theorem 3, equation (16) becomes

$$
\int_{0}^{+\infty} e^{r z} z e^{-z} d z-1=c r
$$

If $r \geq 1$, then

$$
\int_{0}^{+\infty} e^{r z} z e^{-z} d z=+\infty
$$

If $r<1$, then we have

$$
\frac{1}{(1-r)^{2}}-1=c r
$$

or

$$
r\left[c r^{2}-(2 c-1) r+(c-2)\right]=0 .
$$

Since $\rho>0$, it follows that $c>2$. Hence

$$
R=\frac{2 c-1-\sqrt{(2 c-1)^{2}-4 c(c-2)}}{2 c}=\frac{2 c-1-\sqrt{4 c+1}}{2 c}
$$

is the unique solution of equation (17) satisfying $0<R<1$. For $c=$ $2.1,2.2$ and 2.4 , we have $R=0.03191,0.06125$ and 0.11338 respectively. $\Psi(u)$ is strictly decreasing in $R$ for fixed $u>0$, it is suffice to examine $R=0.03191$. So if $u>500$, then by (15), the ruin probability

$$
\Psi(u) \leq e^{-0.03191 \times 500}=1.1771 \times 10^{-7}
$$

is sufficiently small. So if $\inf \{t: R(t)>500\}<\inf \{t: R(t)<0\}$, we classify the company as "non-ruin". Otherwise, if $\inf \{t: R(t)<0\}<$ $\inf \{t: R(t)>500\}$, we consider the company as "ruin". In other words, in 
our example, "non-ruin" means that the time for the total capital exceeding 500 comes prior to the time the total capital being less than 0 . This assumption is reasonable because once the total capital exceeds 500, the chance of "ruin" is slim.

We consider the hypothesis test problem $H_{0}: \Phi(u)=P$. If $H_{0}$ is true, then $S=E \sqrt{R+N R} / \sqrt{P(1-P)}$ has approximately a standard normally distribution, $P(-1.96 \leq S \leq 1.96)=0.95$. (Note that $\sqrt{(R+N R)}=$ $\sqrt{\left(10^{6}\right)}=1000$ in Example 1, while in Example 2, $\sqrt{(R+N R)}=\sqrt{\left(10^{4}\right)}=$ 100.) The simulation results show that the relative frequency of ruin (i.e., F) is very close to the probability of ruin computed by Theorem 3 (i.e., P), and all $S$ values fall in the interval $[-1.96,1.96]$. These results confirm that Theorem 3 is correct.

\section{Concluding Remarks}

The computation of a ruin probability is in many cases difficult. This paper gives a way of computing the ruin probability in a special case. Although the result of Theorem 1 is for gamma distributions with $n$ being positive integer, we are currently only able to give a complete solution for the case of $n=2$. We have obtained solutions for the characteristic equations corresponding to equation (1) in Theorem 1 for $n=3$ and 4 . The computation of the ruin probabilities for these cases is ongoing.

\section{References}

1. Grandell, J., (1991) Aspects of Risk Theory. Springer-Verlag, New York.

2. S. Lang, (1979) Calculus of several variables, secondedition. Addison-Wesley Publishing Company, Massachusetts.

3. Thorin, O., (1973) The ruin problem in case the tail of the claim distribution is completely monotone. Skand. Aktuar Tidskr., 100-119. 


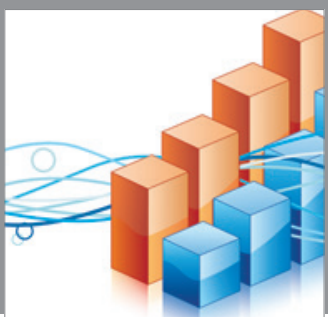

Advances in

Operations Research

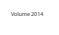

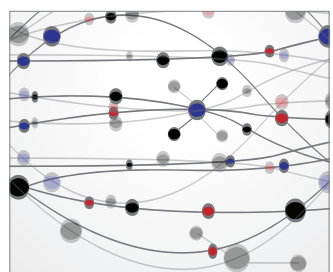

\section{The Scientific} World Journal
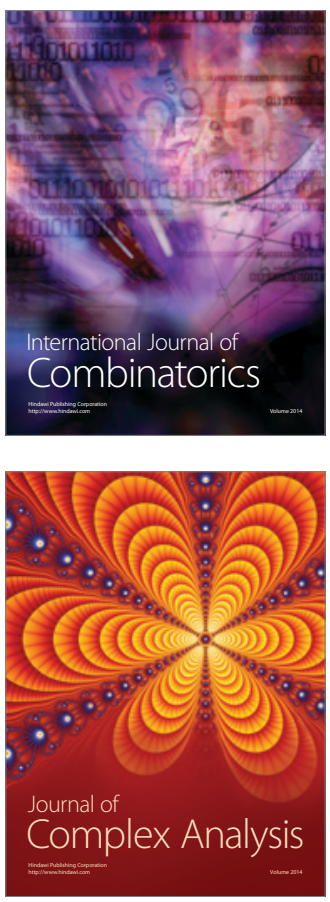

International Journal of

Mathematics and

Mathematical

Sciences
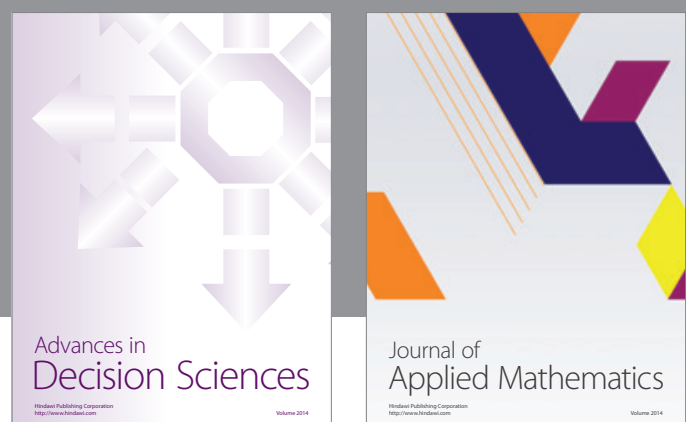

Journal of

Applied Mathematics
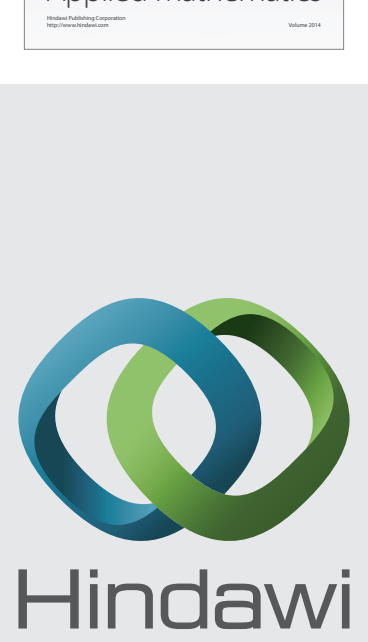

Submit your manuscripts at http://www.hindawi.com
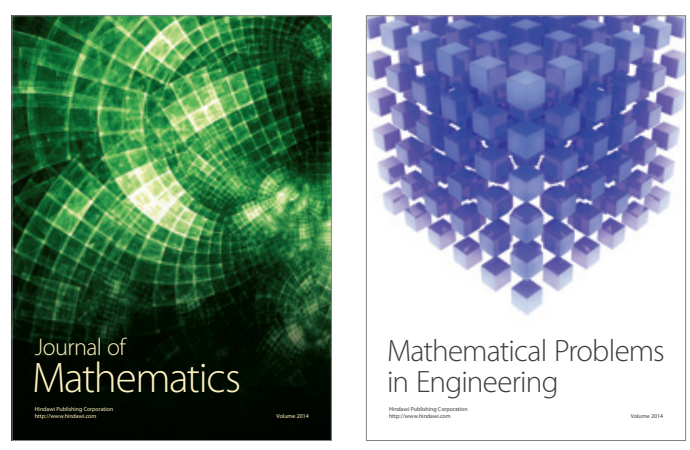

Mathematical Problems in Engineering
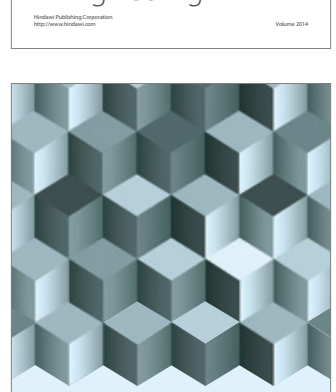

Journal of

Function Spaces
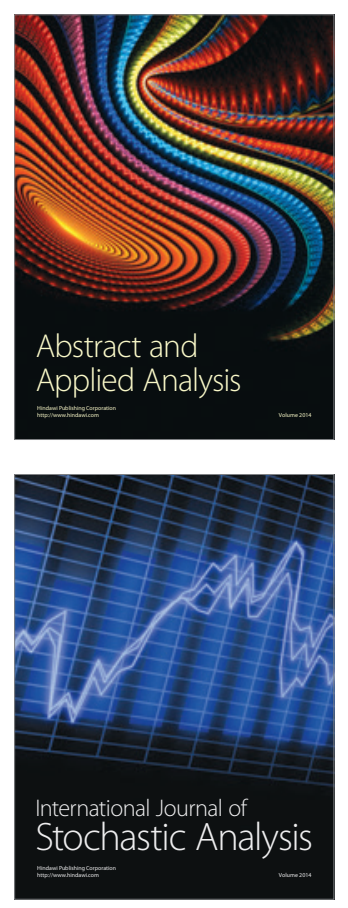

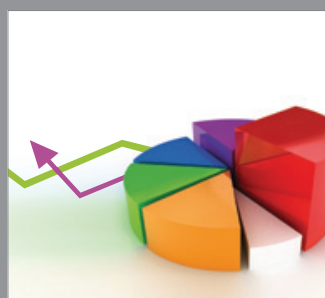

ournal of

Probability and Statistics

Promensencen
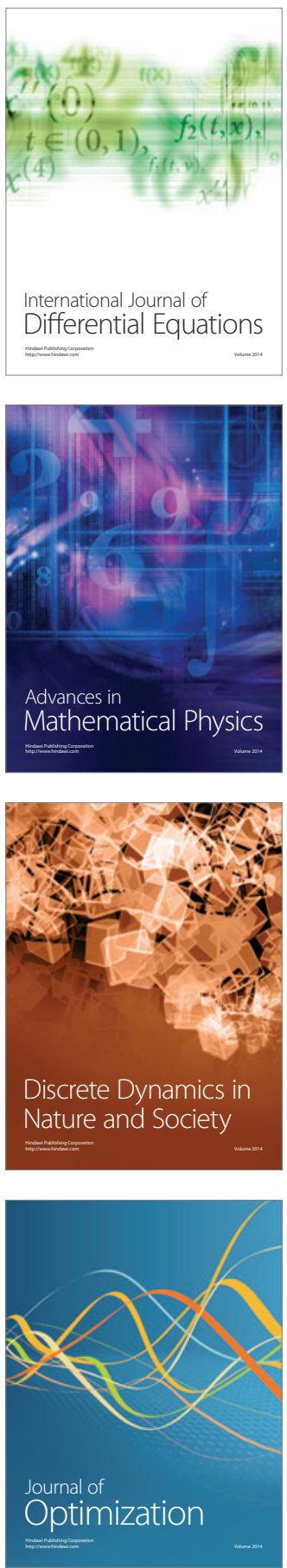